\title{
Characterization and Antifungal Susceptibility Profile of Candida Species Isolated from Candiduria Cases in a Tertiary Care Hospital, Kolkata
}

\author{
Reena Ray-Ghosh $^{{ }^{*}}$, Piyali Haldar ${ }^{2}$ and Mitali Chatterjee ${ }^{1}$ \\ ${ }^{1}$ Department of Microbiology, R.G. Kar. Medical College \& Hospital, Kolkata, India \\ ${ }^{2}$ Department of Microbiology, Govt. Medical College, Diamondharbour, Kolkata, India \\ *Corresponding author
}

\begin{tabular}{|l|}
\hline Ke y w o r d s \\
Antifungal \\
susceptibility \\
profile, Candida \\
Species, Candiduria \\
\hline Article Info \\
\hline $\begin{array}{l}\text { Accepted: } \\
\text { 10 May 2019 } \\
\text { Available Online: } \\
\text { 10 June } 2019\end{array}$ \\
\hline
\end{tabular}

Fungal infections of the urinary tract encompass a broad variety of fungi, However,

\section{Introduction}

A B S T R A C T

Candida species are unusual cause of urinary tract infections in healthy individuals, but common in hospital setting or among patients with predisposing factors. The isolation of Candida from urine is challenging both for clinician and microbiologist as to whether the candiduria represents colonization or urinary tract infection. Species identification of Candida is also important as non albicans Candida are on rise in recent years which are more resistant to antifungal drugs and as a result morbidity and mortality is also increasing. The objective of this study was to determine the prevalence of Candida species causing UTI, analyze various risk factors associated with candiduria and their antifungal susceptibility pattern. A total of 1860 urine samples from clinically suspected cases of urinary tract infection were analysed in a tertiary care Hospital during a period of one year. 105 Candida isolates were obtained from these urine samples and species identification was done following standard laboratory protocol. Antifungal susceptibility testing was performed by disc diffusion method following CLSI guideline M-44A. Among all suspected cases of urinary tract infection, candiduria was diagnosed in $5.64 \%$ cases. Candida tropicalis 44(42\%) was the predominant isolate followed by Candida albicans$38(36 \%)$, Candida glabrata-14(13\%), Candida parapsilosis-07(07\%) and Candida krusei$02(02 \%)$. The antifungal susceptibility pattern showed that Candida isolates were more resistant to Fluconazole-39(37\%), followed by Itraconazole-30 (28.5\%). Fluconazole resistance was more common among non albicans Candida (41\%) than Candida albicans (29\%). Our study reflects a change in trend with shift towards non-albicans candida replacing Candida albicans as the predominant cause of candiduria. Non albicans Candida species are more resistant to antifungal drugs compared to C. albicans. Therefore species identification of Candida isolates along with their antifungal susceptibility pattern is important to guide clinicians for better therapeutic options which will reduce both morbidity as well as financial burden on patients. the overwhelming majority of fungal infections of the urinary tract are caused by Candida species. Candida species account for almost $10-15 \%$ nosocomial UTI ${ }^{1}$. The 
important risk factors associated with candiduria are anatomic defects of urinary tract, indwelling urinary drainage devices, abdominal surgery, ICU stay, broad spectrum antibiotic therapy, diabetes mellitus, increased age and female sex ${ }^{2}$.

Isolation of Candida species from urine samples moves both the Microbiologist and the physician with a challenge as to whether the candiduria represents colonization or, lower or upper urinary tract infection and renal candidiasis with sepsis. ${ }^{3}$

Most observational studies of candiduria have been reported predominance of $C$. albicans ${ }^{3}$. But in recent years with the advent and increasing use of fluconazole, the emergence of non-albicans Candida (NAC) spp. is noted $^{4}$. The NAC spp. are not only well adapted to the urinary tract but also are difficult to eradicate than $C$. albicans. ${ }^{2}$ Therefore, Species identification of Candida is important as non albicans Candida species are increasing in number, more resistant to antifungal drugs and some non-albicans Candida spp. are inherently resistant to treatment with fluconazole. 5,6

The present study was carried out to determine the prevalence of candiduria, phenotypic characterization of the isolated Candida species with their antifungal susceptibility pattern and also to analyse various risk factors associated with candiduria in a tertiary care hospital.

\section{Materials and Methods}

The present study was carried out in the department of Microbiology, in a tertiary care Hospital, Kolkata from over a period of one year. A Total of 1860 urine samples were analyzed. Permission from the institutional ethical committee was taken.

\section{Inclusion criteria}

Both indoor and outdoor patients who had presented with signs and symptoms of urinary tract infection (bladder discomfort, low grade fever, and leucocytosis) were included in the study. Pure growth of yeast isolates with significant colony count was included in the study.

\section{Exclusion criteria}

The urine samples where Candida species was isolated in the absence of pyuria, Candida with colony count $\leq 1000 \mathrm{CFU} / \mathrm{ml}$ and mixed growth were excluded from analysis.

The mid-stream clean-catch urine samples were collected aseptically in a sterile container and transported immediately to microbiology laboratory. The urine samples were inoculated on Blood agar and Cysteine Lactose Electrolyte Deficient media (CLED) by semi-quantitative method as per standard protocol for urine culture. The culture plates were incubated aerobically at $37^{\circ} \mathrm{C}$ for 24 to 48 hours. Candida species isolated on culture plates with colony count $>10000 \mathrm{CFU} / \mathrm{ml}$ were considered significant. Contamination was differentiated from infection by obtaining second urine sample.

The 105 Candida isolates were further speciated by conventional phenotypic methods i.e. Gram staining, culture on Sabouraud's dextrose agar, germ tube test, growth on corn meal agar, sugar fermentation, sugar assimilation test and colony colour in Chrome agar. Antifungal susceptibility testing was performed by disc diffusion method according to CLSI guideline M44A.The antifungal drugs tested were AmphotericinB, Ketoconazole, Fluconazole, Itraconazole, and Voriconazole. 
The antifungal discs were procured from Himedia Laboratories Pvt. Limited, Mumbai. Muller-Hinton agar supplemented with $2 \%$ glucose and $0.5 \mu \mathrm{g} / \mathrm{ml}$ methylene blue was used for the sensitivity testing. C. albicans (ATCC 90028) and C. krusei (ATCC 6258) were used as control strains.

Patient's demographic and clinical data were recorded in a proforma.

\section{Results and Discussion}

A total of $105(5.6 \%)$ samples showed the growth of Candida species out of 1860 urine samples.

In the study candiduria was found to be more common in females- $56(53 \%)$ than males- 49 (47\%). In both the sexes maximum patients belong to age group $>60$ years

In most cases association of more than one risk factor was observed. Broad spectrum antibiotics use was found to be the most important risk factor. Out of 105 patients $82(78 \%)$ were on broad spectrum antibiotics. Urinary catheterization $60(57 \%)$ and diabetes mellitus -44 (42\%), pregnancy $(23 \%)$ were the other major risk factor noted for the development of candiduria.

Out of 105 isolates, Candida tropicalis $44(42 \%)$ were found to be the most common isolate followed by Candida albicans 38(36\%), Candida glabrata 14(13\%), Candida parapsilosis 7(07\%) and Candida krusei-2(02\%)

Table 1 shows antifungal sensitivity pattern of Candida isolates. Maximum resistance was seen against fluconazole-39 out of 105 isolates $(37 \%)$. Fluconazole resistance was more in Non albicans Candida i.e. Candida krusei-2(100\%), Candida glabrata 10 (72\%), Candida tropicalis $-14(32 \%)$ than C. albicans
11(29\%). Amphotericin B \& Voriconazole resistance was seen only in $5.7 \%$ and $3.8 \%$ isolates respectively. Hence irrespective of any species of Candida isolate, the most effective drug can be considered as voriconazole (98\%) followed by Amphotericin-B (96\%) and Ketoconazole $(77 \%)$

Candida species is considered as the fifth most common nosocomial pathogen in India ${ }^{7}$. In our study the prevalence of candiduria is $5.64 \%$ while Anju et al., documented candiduria cases as $1.3 \%$ among all patients with urinary tract infection ${ }^{8}$. However in the literature it has been documented as $11-52 \%$ cases of nosocomial urinary tract infections are caused by Candida species $^{9-11}$.

In the present study, a shift towards NAC (Non-albicans Candida) spp. replacing Candida albicans as a causative agent of candiduria was noted. Candida tropicalis (42\%) was the major isolate in our study. Contributory factors in the emergence of $C$. tropicalis includes injudicious use of antifungal drugs, increase in the population of immunocompromised hosts, use of broad spectrum antibiotics and long term use of catheters $^{12}$.

Study of Prasad et al., also showed that isolation of non- Candida albicans spp. was higher $(52.4 \%)$ as compared to $C$. albicans $(47.6 \%)^{13}$. Chakrabarthi A et al found $C$. tropicalis $(58.7 \%)$ was the commonest species isolated from urine followed by $C$. albicans $^{14}$.

In our study Candiduria was found to be more common in female $(53 \%)$ than male $(47 \%)$ patients. Similar observation was seen in the study done by Payam Behzadi et al., ${ }^{15}$. Since colonization of vulvo vestibular area with Candida spp. is frequent in females, they are more at risk of developing candiduria due to 
ascending infection ${ }^{1}$. In the present study, we also found that UTI due to Candida spp. was more common in old age (>60 yrs). This could be due to lowered host defence at extremes of age.

In the present study more than one risk factor was identified in patient with candiduria. The most common associated risk factor was long term antibiotic therapy (78\%). Similar finding was observed by study of Weinberger et al., ${ }^{16}$. It is likely that antibiotics contribute to colonization by Candida species by suppressing endogenous bacterial flora ${ }^{17}$.
Urinary catheterization was found to be the second most important risk factor in our study. Guler et al., ${ }^{18}$ reported that the risk of development of candiduria is increased 12fold after use of urinary catheter. This may be due to the fact that Catheters placed into the bladder serve as a portal of entry for microorganisms into the urinary drainage system and become colonized if left in place long enough. ${ }^{19}$ Sebastian et al., also studied an association of candiduria with disseminated candidiasis, diabetes mellitus, pregnancy, long term use of broad spectrum antibiotics and the use of catheters ${ }^{8}$.

Table.1 Antifungal susceptibility pattern of different Candida species

\begin{tabular}{|c|c|c|c|c|c|c|}
\hline Antifungal drugs & $\begin{array}{l}\text { Candida } \\
\text { tropicalis } \\
(\mathrm{n}-44)\end{array}$ & $\begin{array}{l}\text { Candida } \\
\text { albicans } \\
(\mathbf{n}-38)\end{array}$ & $\begin{array}{l}\text { Candida } \\
\text { glabrata } \\
(\mathrm{n}-14)\end{array}$ & $\begin{array}{l}\text { Candida } \\
\text { parapsilosis } \\
(\mathbf{n}-7)\end{array}$ & $\begin{array}{l}\text { Candida } \\
\text { krusei } \\
(\mathbf{n}-2)\end{array}$ & \\
\hline \multicolumn{7}{|l|}{ Amphotericin B } \\
\hline Sensitive & $40(91 \%)$ & $34(89 \%)$ & $13(93 \%)$ & $7(100 \%)$ & $2(100 \%)$ & $96(91.2 \%)$ \\
\hline $\begin{array}{l}\text { Dose-dependent } \\
\text { Sensitive }\end{array}$ & $1(2 \%)$ & $2(5 \%)$ & - & - & - & $3(2.8 \%)$ \\
\hline Resistant & $3(7 \%)$ & $2(5 \%)$ & $1(7 \%)$ & _- & _- & $6(5.7 \%)$ \\
\hline \multicolumn{7}{|l|}{ Fluconazole } \\
\hline Sensitive & $24(54 \%)$ & $25(66 \%)$ & $2(14 \%)$ & $5(71 \%)$ & _- & $56(53.3 \%)$ \\
\hline $\begin{array}{l}\text { Dose-dependent } \\
\text { Sensitive }\end{array}$ & $6(14 \%)$ & $2(5 \%)$ & $2(14 \%)$ & - & - & $10(9.5 \%)$ \\
\hline Resistant & $14(32 \%)$ & $11(29 \%)$ & $10(72 \%)$ & $2(29 \%)$ & $2(100 \%)$ & $39(37 \%)$ \\
\hline \multicolumn{7}{|l|}{ Itraconazole } \\
\hline Sensitive & $27(61 \%)$ & $26(68 \%)$ & $3(21 \%)$ & $5(71 \%)$ & $1(50 \%)$ & $62(59 \%)$ \\
\hline $\begin{array}{l}\text { Dose-dependent } \\
\text { Sensitive }\end{array}$ & $5(11 \%)$ & $3(8 \%)$ & $4(29 \%)$ & $1(14 \%)$ & - & $13(12 \%)$ \\
\hline Resistant & $12(27 \%)$ & $9(24 \%)$ & $7(50 \%)$ & $1(14 \%)$ & $1(50 \%)$ & $30(28.5 \%)$ \\
\hline \multicolumn{7}{|l|}{ ketoconazole } \\
\hline Sensitive & $32(73 \%)$ & $27(71 \%)$ & $11(79 \%)$ & $5(71 \%)$ & $2(100 \%)$ & $77(73 \%)$ \\
\hline $\begin{array}{l}\text { Dose-dependent } \\
\text { Sensitive }\end{array}$ & $3(7 \%)$ & $2((5 \%)$ & $1(7 \%)$ & $1(14 \%)$ & - & $7(6.6 \%)$ \\
\hline Resistant & $9(20 \%)$ & $9(24 \%)$ & $2(14 \%)$ & $1(14 \%)$ & _- & $21(20 \%)$ \\
\hline \multicolumn{7}{|l|}{ Voriconazole } \\
\hline Sensitive & $40(91 \%)$ & $36(95 \%)$ & $13(93 \%)$ & $7(100 \%)$ & $2(100 \%)$ & $98(93.3 \%)$ \\
\hline $\begin{array}{l}\text { Dose-dependent } \\
\text { Sensitive }\end{array}$ & $2(4.5 \%)$ & $1(2.5 \%)$ & - & - & - & $3(2.8 \%)$ \\
\hline Resistant & $2(4.5 \%)$ & $1(2.5 \%)$ & $1(7 \%)$ & _ & _ & $4(3.8 \%)$ \\
\hline
\end{tabular}


Fig.1 Age and sex distribution of patients with candiduria

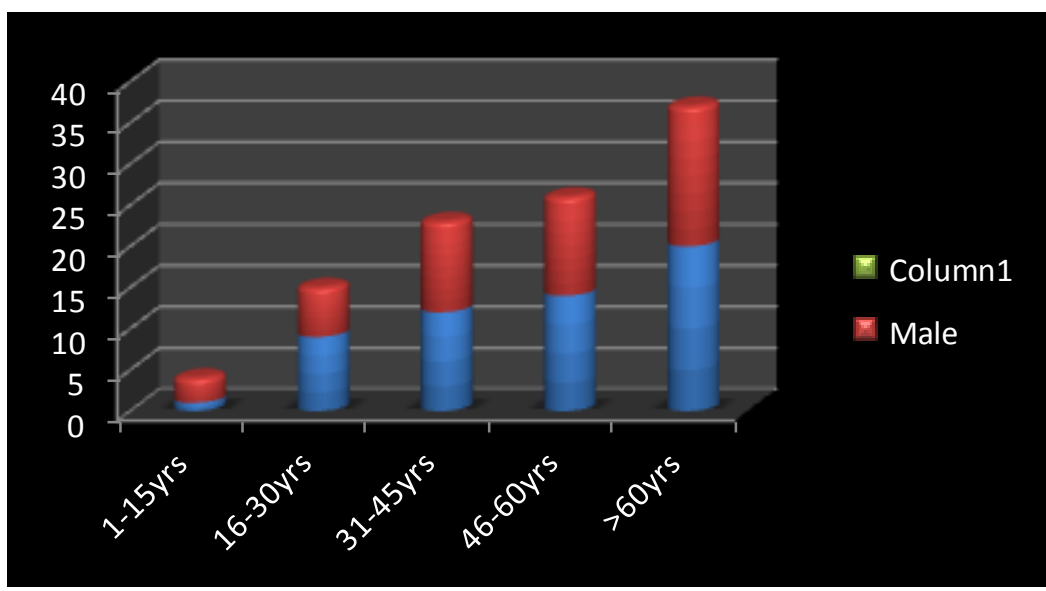

Fig.2 Associated risk factors

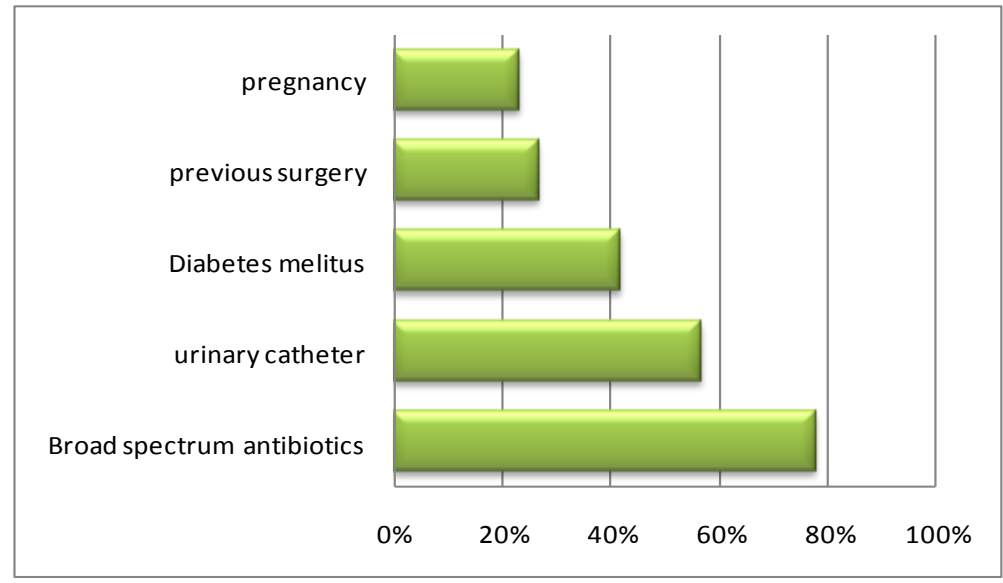

Fig.3 Distribution of different Candida species

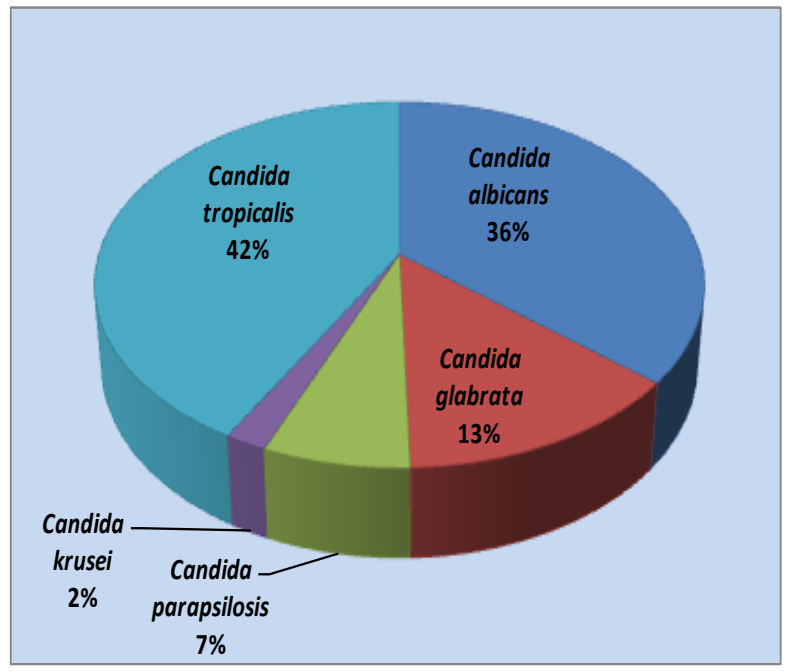


It has been studied and documented in the literature as the risk of candiduria increases by twelve fold following use of urinary catheters, while six, four' two and one fold rise is observed after the use of broad spectrum antibiotics and urinary abnormalities, following abdominal surgeries, in presence of diabetes mellitus and in association with corticosteroid administration respectively ${ }^{3}$.

Antifungal susceptibility pattern in our study showed that Candida isolates developed more resistance against Fluconazole $(37 \%)$ as compared to other antifungal agents. Resistance to Fluconazole was more in the non-Candida albicans group $(\mathrm{p}<0.05)$ when compared to $C$. albicans. Similar results have been observed in the study conducted by Malhotra Aanchal ${ }^{20}$ et al., \& by Omprakash Bobade et al., ${ }^{21}$ from India.

In conclusion, isolation of Candida from urine can no longer be ignored as contamination or commensal. But as the line between colonization, contamination and infection is not very well demarcated the approach to patient with candiduria needs to be individualized. Traditionally Fluconazole is preferred by clinicians to combat any type of fungal infection because of its wider spectrum, affordability, easy to administer and less toxicity. The emergence of nonalbicans Candida as a causative agent of Candiduria and its resistance to Fluconazole has generated great concern. Although in this study Candida species are found to be highly susceptible to Amphotericin and Voriconazole, the situation should be properly weighed before administering these drugs considering its adverse effects and high cost which might put financial burden on the patient. Therefore speciation of Candida and their antifungal susceptibility pattern is crucial for the treatment and to prevent the emergence of drug resistance.

\section{References}

1. Lundstrom T, Sobel J. Nosocomial candiduria: A review. Clin Infect Dis. 2001; 32:1602-07.

2. Sobel JD, Fisher JF, Kauffman CA, Newman CA. Candida urinary tract infection-Epidemiology. Clin In $\mathrm{f}$ e $\mathrm{c} t$ Dis 2011; 52(suppl 6): S433-S436.

3. Bukhary, Z.A. 2008. Candidiuria: A review of clinical significance and its importance. Saudi J Kidney Dis Transplant, 19(3):350-360

4. Achkar JM, Fries BA. Candida infections of the genitourinary tract. Clin Microbiol Rev 2010; 23: 253-73.

5. Hartstein AI, Garber SB, Ward TT, Jones SR, Morthland VH. Nosocomial urinary tract infection: a prospective evaluation of 108 catheterized patients. Infect. Control. 1981; 2: 380-6.

6. Annaissie E, Samonis G, Kontoyiannis D, Costerton J, Sabharwal U, Bodey G et al., Role of catheter related colonization and infrequent hematogenous seeding in catheter related infections. Eur J Clin Microbiol Infect Dis. 1995; 14: 135-7.

7. Prasad KN, Agarwal J, Dixit AK, Tiwari DP, Dhole TN, Ayyagiri A. Role of yeast as nosocomial pathogens and their susceptibility to Fluconazole and Amphotericin. Ind. J Med. Res. 1999; 110: 11-17.

8. Sebastian A, Archana S. Species identification and antifungal susceptibility of Candida isolated from urine specimens in a tertiary care hospital. IP Journal of Medical Microbiology and Tropical Diseases. 2018; 4(2): 7375. 
9. Richards MJ, Edwards JR, Culver DH. Nosocomial infections in combined medical-surgical intensive care units in the United States. Infect Control Hosp Epidemiol. 2000; 21:510-5.

10. Brindha S, Jayashree M, Singhi S. Study of nosocomial urinary tract infections in a paediatric intensive care unit. J Trop Pediatr. 2011; 57: 357-62.

11. Rivett A, Perry J, Cohen J. Urinary candidiasis: a prospective study in hospital patients. Urol Res 1986; 14: 183-6.

12. Kothavade RJ, Kura MM, Valand AG, Panthaki MH. Candida tropicalis: its prevalence, pathogenicity and increasing resistance to fluconazole. J Med Microbiol 2010; 59(8): 873-80.

13. Prasad KN, et al., Role of yeasts as nosocomial pathogens and their susceptibility to Fluconazole and Amphotericin. Ind $\mathbf{J}$ of Med Res. 1999; 110:11-17.

14. Chakrabarthi A, Reddy TCS, Singhi S. Does Candiduria predict candidaemia? Ind $\mathbf{J}$ of Med Res. 1997; 106; 513-16.

15. Payam Behzadi, Elham Behzadi, Hodjjat Yazaanbod. Urinary tract infections associated with Candida albicans. $\mathrm{J}$ of medicine. maedica, dec 2010: 5(4): 277-279.

16. Weinberger M, Sweet S, Leiboviciy L, Pitlik SD, Samraz Z. Correlation between candiduria and departmental antibiotic use. J Hosp Infect. 2003; 53: 183-6.

17. Fisher JF, Chew WH, Shadomy S, Duma RJ, Mayhall CG, House WC. Urinary tract infections due to Candida albicans. Rev Infect Dis, 1982; 4: 1107-18.

18. Guler S, Ural O, Findik D, Arslan U. Risk factors for nosocomial candiduria. Saudi Med J 2006; 27(11): 1706-10.

19. Stamm WE. Catheter-associated urinary tract infections: epidemiology, pathogenesis, and prevention. Am J Med 1991; 91(Suppl 3B): 65S-71S.

20. Malhotra A, Gupta V, Chhina k.D, Singh A. Speciation and antifungal susceptibility profile of candiduria cases at a tertiary care Hospital. IJPRBS, 2015; Volume 4(6): 137144.

21. Bobade O, Waghmare M, Chhabrani P, Kaur I. Species distribution and antifungal susceptibility profile of Candida isolated from urine samples. International Journal of Medical Science and Public Health | 2013 | Vol 2 | Issue 4

\section{How to cite this article:}

Reena Ray (Ghosh), Piyali haldar and Mitali Chatterjee. 2019. Characterization and Antifungal Susceptibility Profile of Candida Species Isolated from Candiduria Cases in a Tertiary Care Hospital, Kolkata. Int.J.Curr.Microbiol.App.Sci. 8(06): 1079-1085.

doi: https://doi.org/10.20546/ijcmas.2019.806.133 